\title{
Erratum to: $\mathrm{H}_{2} \mathrm{O}$ storage capacity of olivine and low-Ca pyroxene from 10 to $13 \mathrm{GPa}$ : consequences for dehydration melting above the transition zone
}

Travis J. Tenner • Marc M. Hirschmann •

Anthony C. Withers · Paola Ardia

Published online: 8 September 2011

(C) Springer-Verlag 2011

Erratum to: Contrib Mineral Petrol

DOI 10.1007/s00410-011-0675-7

Unfortunately, Table 5 of the original article was published with incorrect data. The row of $D_{\mathrm{H}_{2} \mathrm{O}}^{\mathrm{pyx} / \mathrm{ol}}$ should read as 1.39 , $1.36,1.37,1.15,1.54$, and 1.28 . The correct version of Table 5 is given below.

The online version of the original article can be found under doi: 10.1007/s00410-011-0675-7.

T. J. Tenner $(\bowtie) \cdot$ M. M. Hirschmann .

A. C. Withers · P. Ardia Department of Geology and Geophysics,

University of Minnesota, 108 Pillsbury Hall,

310 Pillsbury Drive, Minneapolis, MN 55455, USA

e-mail: tenn0047@umn.edu 
Table 5 SIMS-derived $\mathrm{H}_{2} \mathrm{O}$ storage capacities of olivine and low-Ca pyx, partitioning of $\mathrm{H}_{2} \mathrm{O}$ between olivine and low-Ca pyx, and EMPderived mass balance data

\begin{tabular}{|c|c|c|c|c|c|c|c|c|c|c|}
\hline Exp. \# & M455 & M461 & M468 & M474 & M467 & M471 & M476 & M463 & M470 & M465 \\
\hline $\mathrm{P}(\mathrm{GPa})$ & 10 & 11 & 12 & 12 & 12 & 12 & 12 & 12 & 12 & 13 \\
\hline $\mathrm{T}\left({ }^{\circ} \mathrm{C}\right)$ & 1,450 & 1,450 & 1,350 & 1,350 & 1,400 & 1,400 & 1,400 & 1,450 & 1,450 & $1450^{\mathrm{a}}$ \\
\hline Bulk $\mathrm{H}_{2} \mathrm{O}$ (wt. \%) & 1.00 & 0.48 & 0.48 & 1.00 & 0.48 & 0.75 & 1.00 & 0.48 & 0.75 & 0.48 \\
\hline n (ol, opx) & 8 & 8 & 9,3 & 7,3 & 8,3 & 5,2 & 7 & 8 & 7,3 & 8,5 \\
\hline $\begin{array}{l}\text { Olivine storage } \\
\text { cap. (ppm) }\end{array}$ & $\begin{array}{l}410 \\
(60)\end{array}$ & $\begin{array}{l}330 \\
(50)\end{array}$ & $1,140(100)$ & $920(100)$ & $760(70)$ & $\begin{array}{l}1,190 \\
(120)\end{array}$ & $\begin{array}{l}860 \\
(80)\end{array}$ & $600(60)$ & $\begin{array}{l}720 \\
(70)\end{array}$ & $470(100)$ \\
\hline $\begin{array}{l}\text { Low-Ca pyx } \\
\text { storage cap. } \\
(\mathrm{ppm})\end{array}$ & & & $1,580(60)$ & $1,250(60)$ & $1,040(60)$ & $\begin{array}{r}1,370 \\
(50)\end{array}$ & & & $\begin{array}{r}1,110 \\
(80)\end{array}$ & $600(40)$ \\
\hline$D_{\mathrm{H}_{2} \mathrm{O}}^{\mathrm{pyx} / \mathrm{ol}}$ & & & 1.39 & 1.36 & 1.37 & 1.15 & & & 1.54 & 1.28 \\
\hline $\begin{array}{l}\text { Modes (F, Ol, LPx, } \\
\text { HPx, Gt) }\end{array}$ & & & $\begin{array}{l}0.04,0.68,0.09 \\
0.05,0.14\end{array}$ & $\begin{array}{l}0.10,0.62,0.13, \\
0.01,0.13\end{array}$ & $\begin{array}{l}0.09,0.60 \\
0.17,0,0.13\end{array}$ & & & $\begin{array}{c}0.08,0.66 \\
0.12, \text { tr., } \\
0.14\end{array}$ & & $\begin{array}{l}0.07,0.59 \\
0.19,0,0.15\end{array}$ \\
\hline $\mathrm{H}_{2} \mathrm{O}_{\text {melt }}$ (wt. \%) & & & 12 & 10 & 11 & & & 6 & & 7 \\
\hline Rel. Fe loss (\%) & & & 0 & 1.5 & 11 & & & 10 & & 0 \\
\hline$D_{\mathrm{H}_{2} \mathrm{O}}^{\mathrm{ol} / \mathrm{melt}}$ & & & 0.010 & 0.010 & 0.007 & & & 0.010 & & 0.007 \\
\hline$D_{\mathrm{H}_{2} \mathrm{O}}^{\mathrm{pyx} / \mathrm{melt}}$ & & & 0.013 & 0.013 & 0.009 & & & & & 0.009 \\
\hline
\end{tabular}

Errors (in parentheses) are propagated errors, combining the $1 \sigma \mathrm{SD}$ of measurements and the uncertainty in the SIMS calibration (Fig. 6)

${ }^{a}$ Temperature estimated from the power required to heat the experiment 